\section{CURVAS DE ABSORCIÓN DE MACRONUTRIENTES EN CALABACITA ITALIANA (Cucurbita pepo L.)}

\section{MACRONUTRIENTS ABSORPTION CURVES IN ITALIAN SQUASH (Cucurbita pepo L.)}

\author{
Heberto A. Rodas-Gaitán, Humberto Rodríguez \\ -Fuentes*, Ma. del Carmen Ojeda-Zacarías, Juan A. \\ Vidales-Contreras y Alejandro I. Luna-Maldonado
}

Subdirección de Estudios de Posgrado, Facultad de Agronomía, Universidad Autónoma de Nuevo León, Campus de Ciencias Agropecuarias, Av. Francisco Villa s/n, Col. Ex Hacienda El Canadá. 66050, Municipio General Escobedo, Nuevo León, México.

*Autor para correspondencia (hrodrigu10@yahoo.com.mx)

\section{RESUMEN}

Mediante análisis químicos se determinaron las curvas de crecimiento y de absorción de algunos nutrimentos esenciales durante el ciclo de producción de la calabacita italiana (Cucurbita pepo L.), en un lote de producción comercial. La producción de biomasa seca total fue de 243 $\mathrm{g}$ /planta. Los datos de absorción de nitrógeno $(\mathrm{N})$, fósforo $(\mathrm{P})$, potasio $(\mathrm{K})$, calcio $(\mathrm{Ca})$ y magnesio $(\mathrm{Mg})$ a través del tiempo se ajustaron a modelos lineales de regresión, cuyos coeficientes de determinación $\left(\mathbf{R}^{2}\right)$ fueron: de 0.89, 0.92, 0.94, 0.78 y 0.72 para $\mathrm{N}, \mathrm{P}, \mathrm{K}$, Ca y $\mathrm{Mg}$, respectivamente. De acuerdo con la densidad de siembra (26 600 plantas ha $\left.^{-1}\right)$, la absorción total (g/planta) de cada mineral fue: $6.75 \mathrm{~N}, 0.67 \mathrm{P}, 1.37 \mathrm{~K}$, 7.47 Ca y $2.07 \mathrm{Mg}$.

Palabras clave: Cucurbita pepo, etapa fenológica, regresión lineal.

\section{SUMMARY}

Chemical analyzes were conducted to determine plant growth and absorption curves for some essential nutrients during the Italian squash (Cucurbita pepo L.) growing season in a commercial production plot. The total dry biomass production was $243 \mathrm{~g} / \mathrm{plant}$; time series absorption data for nitrogen $(\mathrm{N})$, phosphorus $(\mathrm{P})$, potassium $(\mathrm{K})$, calcium $(\mathrm{Ca})$, and magnesium $(\mathrm{Mg})$ were adjusted to linear models; the regression coefficients $\left(R^{2}\right)$ were: $0.89,0.92,0.94,0.78$ and 0.72 for $N$, $\mathrm{P}, \mathrm{K}, \mathrm{Ca}$, and $\mathrm{Mg}$, respectively. According to plant population (26 600 plants ha ${ }^{-1}$ ), the total absorption ( $\mathrm{g} /$ plant) for each mineral was: $6.75 \mathrm{~N}$, 0.67 P, 1.37 K, 7.47 Ca, and 2.07 Mg.

Index words: Cucurbita pepo, phenological stage, linear regression.

\section{INTRODUCCIÓN}

La nutrición es un factor que influye directamente en la producción del cultivo de calabacita (Cucurbita pepo L.), en el que se deben considerar aspectos como la época de crecimiento y el método y lámina de riego a aplicar, los cuales afectan significativamente la producción y la calidad del fruto. La interacción entre etapa de crecimiento, método de aplicación de fertilizantes, lámina de riego y $\mathrm{N}$ disponible, afecta significativamente el índice de área foliar, los sólidos solubles totales, la producción de biomasa seca, y al número y peso de frutos (Jamil Mohammad, 2004a; Zotarelli et al., 2008; Amer, 2011).

La tecnología generada en los últimos años ha tendido a hacer más eficientes y sustentables los sistemas de producción agrícola, con la finalidad de disminuir costos, incrementar la rentabilidad, ofertar alimentos inocuos y disminuir los impactos negativos al ambiente que resultan de la excesiva aplicación de agroquímicos. En este sentido, una alternativa útil es estimar la demanda nutrimental para cada especie cultivada con base en el concepto de balance de masa. Éste se basa en que la materia seca de una planta está formada por 16 elementos químicos esenciales, de los cuales 13 se adicionan directamente al suelo para ser absorbidos por la planta por medio de las raíces. Con la cantidad de materia seca y concentración de cada uno de los elementos en cada etapa fenológica del cultivo, se puede estimar la cantidad total de cada nutrimento que la planta absorbió. Sin embargo, debe considerarse tanto la eficiencia de absorción de los nutrimentos como la aplicación de la fuente fertilizante, para luego establecer un plan de fertilización que podría ser diario, semanal, quincenal, etc. (EtcheversBarra, 2000; Alpízar et al., 2006; Rodríguez-Fuentes et al., 2012).

Es entonces de importancia generar información de la tasa de absorción nutrimental por especie y sus condiciones ambientales, para cada sistema de producción (Magnífico et al., 1979; Honorato et al., 1993; Scaife y Bar-Yosef, 1995). Debido a la especificidad de las condiciones locales de producción, existe escasa información que guíe a los productores con respecto a los requerimientos nutricionales del cultivo, lo cual conlleva al uso de fórmulas de fertilización generales que no contemplan las necesidades específicas del cultivo ni las características de suelo (Alpízar et al., 2006).

Los objetivos de este ensayo fueron: 1) Estimar la curva de crecimiento y absorción de N, P, K, Ca y Mg en calabacita italiana en un sistema de producción en campo; y 2) Generar modelos de regresión de su crecimiento y absorción nutrimental.

\section{MATERIALES Y MÉTODOS}

La investigación se llevó a cabo en Viñedos Llanos de San Francisco, H. Caborca, Sonora, México ( $30^{\circ} 50^{\prime} 21^{\prime \prime} \mathrm{LN}$, $\left.112^{\circ} 45^{\prime} 17^{\prime \prime} \mathrm{LO}\right)$, a $81 \mathrm{msnm}$. El trabajo experimental se desarrolló entre el 27 de febrero y el 17 de mayo del 2006. Para el establecimiento del cultivo se utilizó semilla híbrida del cultivar 'Contender'. La siembra se hizo a doble hilera, 
a una distancia de $0.3 \mathrm{~m}$ entre plantas, $0.3 \mathrm{~m}$ entre hileras (doble) y $2.5 \mathrm{~m}$ entre surcos en una superficie de 20 ha.

La aplicación de fertilizante fue a través de un sistema de fertirriego. Las cantidades totales de fertilizantes (en dosis/ha) aplicadas al suelo durante el ciclo fueron: $290 \mathrm{~kg}$ $\mathrm{CO}\left(\mathrm{NH}_{2}\right)_{2}, 455 \mathrm{~kg} \mathrm{Ca}\left(\mathrm{NO}_{3}\right)_{2}, 300 \mathrm{~kg} \mathrm{P} \mathrm{O}_{5}, 155 \mathrm{~L} \mathrm{H}_{3} \mathrm{PO}_{4}, 210$ $\mathrm{kg} \mathrm{KNO}, 4 \mathrm{~L} \mathrm{CaO}$ (foliar), $150 \mathrm{~kg} \mathrm{Mg}\left(\mathrm{NO}_{3}\right)_{2}, 5 \mathrm{~L} \mathrm{~B}$ (foliar) y $2.25 \mathrm{~kg} \mathrm{~B}$.

\section{Análisis de suelo}

Previo al establecimiento de la plantación, se muestreó el suelo (muestra compuesta) a una profundidad de 0 a 0.35 $\mathrm{m}$. En la muestra se analizaron las características siguientes: $\mathrm{pH}$, materia orgánica, textura, nutrimentos solubles (nitrógeno, fósforo, potasio) (Cuadro 1). Los procedimientos analíticos se realizaron según Rodríguez-Fuentes y Rodríguez-Absi (2011).

\section{Análisis de plantas y curvas de crecimiento}

Posterior a la emergencia se colectaron plantas cada $10 \mathrm{~d}$ hasta finalizar el periodo de producción de fruto (seis muestreos en total). Por cada muestreo se extrajeron tres plantas completas, las cuales fueron representativas de la población (altura, color, sanidad, entre otros) y con completa competencia. En cada muestreo se midió (en g/planta) la biomasa aérea (hojas, tallos, flores, frutos) y de raíz, la producción de biomasa seca total (BS), y los contenidos de N, P, K, Ca y $\mathrm{Mg}$ (en porcentajes sobre base seca).

Para obtener la BS las muestras se secaron en una estufa de convección forzada a una temperatura no mayor a $60{ }^{\circ} \mathrm{C}$ hasta peso constante. Posteriormente las muestras fueron molidas y tamizadas con malla de $0.05 \mathrm{~mm}$.

La determinación de nitrógeno total se hizo con el método de Kjeldhal. Las muestras de fósforo y potasio fueron previamente digeridas en una mufla a una temperatura de 450 a $500{ }^{\circ} \mathrm{C}$ por $6 \mathrm{~h}$. La determinación de la concentración de fósforo se hizo con un espectrofotómetro Spectronic ${ }^{\circledR}$, modelo Helios Epsilon (USA). El análisis de potasio, calcio y magnesio se hizo por espectroscopía de absorción atómica (UNICAM Solar ${ }^{\circledR}$, modelo 9626) (Rodríguez-Fuentes y Rodríguez-Absi, 2011).

Los datos de BS y absorción de nutrimentos con respecto al tiempo fueron ajustados a modelos de regresión mediante el programa SigmaPlot10.0®. Los análisis químicos se efectuaron en el Laboratorio de Suelos, Plantas y Aguas de la Facultad de Agronomía de la Universidad Autónoma de Nuevo León.

\section{RESULTADOS Y DISCUSIÓN}

Los resultados de absorción total de nutrientes (g/planta) fueron: $6.75 \mathrm{~N}, 0.67 \mathrm{P}, 1.37 \mathrm{~K}, 7.47 \mathrm{Ca}$ y $2.07 \mathrm{Mg}$. Se reporta en la literatura que la absorción de nutrientes en C. pepo durante el ciclo del cultivo ocurre con una tendencia lineal positiva, con una absorción total de 1.65, 0.24, 2.66, 2.25 y $0.62 \mathrm{~g} /$ planta para N, P, K, Ca y Mg, respectivamente (Com. pers. $)^{1}$. Tales resultados son menores a los encontrados en nuestro ensayo, con excepción de la absorción de K que fue mayor. Sin embargo, en la misma especie Jamil Mohammad (2004b) reportó una absorción total de 5.23 y 8.03 g de $\mathrm{N}$ por planta al aplicar al suelo 66 y $132 \mathrm{~kg} \mathrm{ha}^{-1}$ de $\mathrm{N}$ respectivamente, con una densidad de siembra de 16667 plantas $\mathrm{ha}^{-1}$. El valor promedio de los datos encontrados en nuestro

Cuadro 1. Análisis de suelo. Viñedos Llanos de S.F., H. Caborca, Sonora.

\begin{tabular}{llc}
\hline Características & \multicolumn{1}{c}{ Análisis } & Clasificación agronómica \\
\hline $\mathrm{pH}$ & 7.96 & \\
& Arena $86.4 \%$ & Arena \\
Textura & Limo $9.2 \%$ & \\
& Arcilla $4.4 \%$ & Muy bajo \\
Materia orgánica (Método Walkley-Black) & $0.41 \%$ & Pobre \\
Nitrógeno total & $0.08 \%$ & Nivel crítico \\
Fósforo extraíble (Método Olsen modificado) & $7.94 \mathrm{mg} \mathrm{kg}^{-1}$ & Nivel crítico \\
Potasio extraíble (Método Olsen modificado) & $15.8 \mathrm{meq}^{-100 \mathrm{~g}}$ & No salino \\
Sales solubles totales (CE) & $1.21 \mathrm{mS} \mathrm{cm}{ }^{-1} \mathrm{a} 25^{\circ} \mathrm{C}$ &
\end{tabular}

$\overline{\mathrm{CE}}=$ conductividad eléctrica del extracto de saturación.

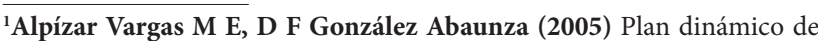
fertilización para escalopine verde (Cucurbita pepo) con los principios de agricultura de precisión. Universidad Earth. Costa Rica. 97 p.
} 


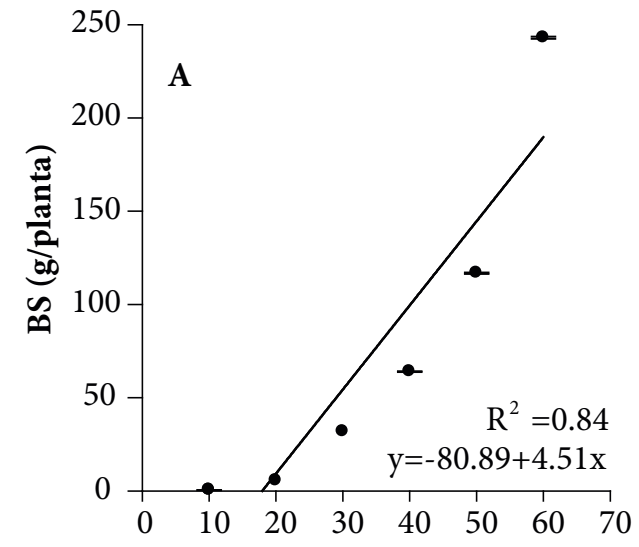

Días después de transplante

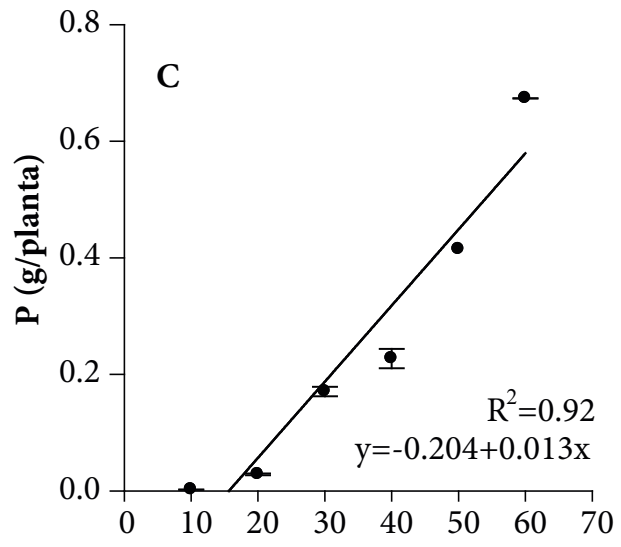

Días después de transplante

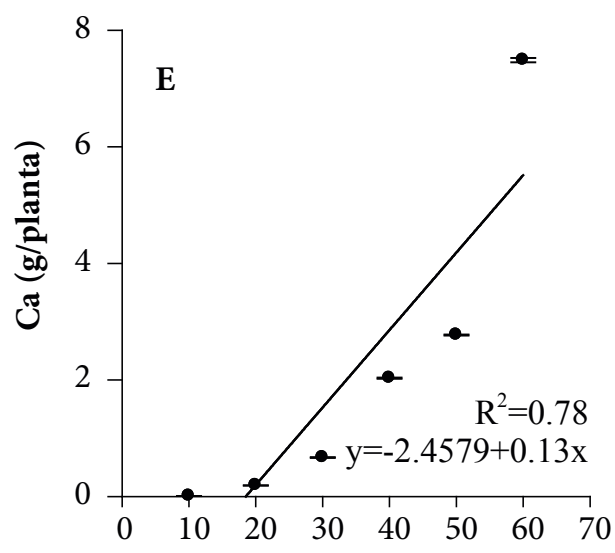

Días después de transplante

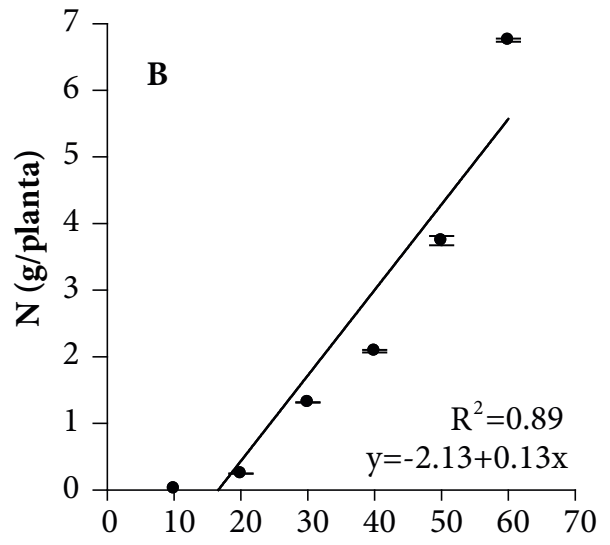

Días después de transplante

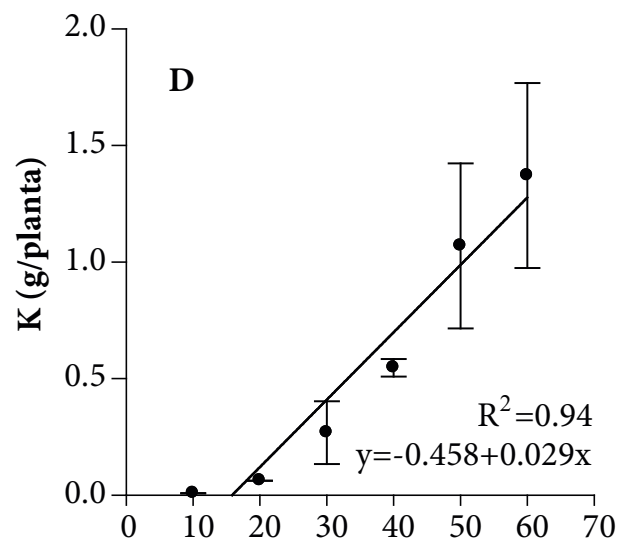

Días después de transplante

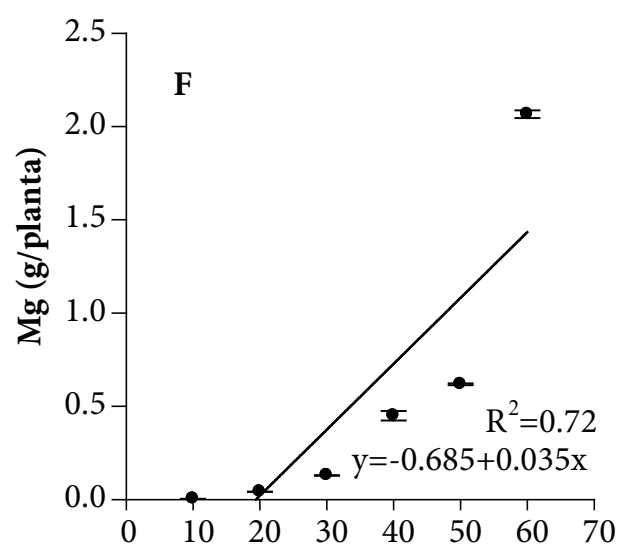

Días después de transplante

Figura 1. Curva de crecimiento de biomasa seca (A), y de absorción de nitrógeno (B), fósforo (C), potasio (D), calcio (E) y magnesio (F) (g/planta, en base seca) durante el ciclo de cultivo, y ajuste a modelos de regresión lineal simple (-). Las barras verticales en cada punto representan la desviación estándar de la media. 
ensayo se encuentra dentro de este rango ( $6.75 \mathrm{~g} \mathrm{~N} /$ planta). El autor citado también menciona que el sistema de fertirrigación generó mayor eficiencia de absorción del fertilizante nitrogenado, lo que es coincidente con nuestro ensayo. La producción de BS fue de $243 \mathrm{~g} /$ planta (Figura 1A), superior al reportado por Colla et al. (2004) cuyo máximo valor fue de $140 \mathrm{~g} /$ planta, con una dosis de $24 \mathrm{~g}$ de $\mathrm{NO}_{3}$ / $/$ planta.

La relación entre la absorción de nutrimentos y tiempo de muestreo se ajustaron a modelos lineales $(\mathrm{P}<0.05$; Figura $1 \mathrm{~B}, 1 \mathrm{C}, 1 \mathrm{D}, 1 \mathrm{E}$ y $1 \mathrm{~F})$. Los valores de $\mathrm{R}^{2}$ fueron: $0.89,0.92$, $0.94,0.78$ y 0.72 para N, P, K, Ca y Mg, respectivamente. Los criterios empleados para seleccionar el mejor modelo fueron el mayor coeficiente de determinación $\left(\mathrm{R}^{2}\right)$ y el menor valor del cuadrado medio residual (CMR), y la mayoría de los modelos para los nutrimentos analizados se ajustaron a estas condiciones. Se considera que el uso de ecuaciones de primer grado estimaran adecuadamente los requerimientos nutricionales del cultivo y con ello se podrá plantear y ejecutar un programa preliminar de fertilización en campo.

\section{CONCLUSIONES}

La curva de crecimiento y la absorción de N, P, K, Ca y $\mathrm{Mg}$ en calabacita italiana se ajustaron a modelos de regresión lineal.

Los modelos de regresión estimados permitirán calcular los requerimientos de $\mathrm{N}, \mathrm{P}, \mathrm{K}, \mathrm{Ca}$ y $\mathrm{Mg}$, y generar un programa de fertilización preliminar propio para la zona y para las condiciones de manejo agronómico donde se efectuó el ensayo.

\section{BIBLIOGRAFÍA}

Alpízar M E, D F González, E Spaans, P Tabora (2006) Plan dinámico de fertilización para escalopine verde (Cucurbita pepo). Tierra Trop. 2:39-47.

Amer K H (2011) Effect of irrigation method and quantity on squash yield and quality. Agric. Water Manage. 98:1197-1206.

Colla G, Y Rouphael, A L Molle, F Saccardo (2004) Influences of mineral nutrition on growth and elemental composition of hydroponically grown zucchini plants. Acta Hort. 644:399-407.

Etchevers-Barra J D (2000) Técnicas de diagnóstico útiles en la medición de la fertilidad del suelo y el estado nutrimental de los cultivos. Terra Latinoam. 17:209-219.

Honorato R, L Gurovich, R Piña (1993) Ritmo de absorción de N, P y K en pepino de semilla. Cien. Invest. Agraria 20:169-172.

Jamil Mohammad M (2004a) Squash yield, nutrient content and soil fertility parameters in response to methods of fertilizer application and rates of nitrogen fertigation. Nutr. Cycling Agroec. 68:99-108.

Jamil Mohammad M (2004b) Utilization of applied fertilizer nitrogen and irrigation water by drip-fertigated squash as determined by nuclear and traditional techniques. Nutr. Cycling Agroec. 68:1-11.

Magnifico V, V Lattancio, G Sarli (1979) Growth and nutrient removal by broccoli. J. Amer. Soc. Hort. Sci. 104:201-203.

Rodríguez-Fuentes H, J Rodríguez-Absi (2011) Métodos de Análisis de Suelo y Plantas. Ed. Trillas S.A. de C.V. México. 239 p.

Rodríguez-Fuentes H, J A Vidales-Contreras, A I Luna-Maldonado, J C Rodríguez-Orti (2012) Total growth of tomato hybrids under greenhouse conditions. In: Horticulture. A I Luna M (ed). InTech. Rijeka, Croatia. pp:63-72.

Scaife A, B Bar-Yosef (1995) Nutrient and Fertilizer Management in Field Grown Vegetables. IPI Bulletin No. 13. International Potash Institute, Basel, Switzerland. $104 \mathrm{p}$.

Zotarelli L, M D Dukes, J M Scholberg, T Hanselman, K L Femminella, R Muñoz-Carpena (2008) Nitrogen and water use efficiency of zucchini squash for a plastic much bed system on a sandy soil. Sci. Hort. 116:8-16. 